\title{
KSR2 mutations affect energy balance, insulin sensitivity and cellular fuel oxidation
}

A new study has identified a range of mutations in KSR2 that are linked to the development of early-onset obesity and severe insulin resistance.

Previous work has shown that deletion of $K s r 2$ leads to obesity in mice, which suggests that it has a role in energy homeostasis. A team of researchers therefore decided to determine whether KSR2 is involved in obesity in humans.

DNA samples were obtained from 2,101 individuals (adults and children) from the Genetics of Obesity Study cohort who had early-onset obesity and 1,536 people from the ELY Study (a sample of the general population from Ely, Cambridgeshire, UK) as controls. The researchers then sequenced the coding region and intronexon boundaries of KSR2. The analysis revealed 27 different variants, which were present in $2.1 \%$ of patients with severe obesity compared with $1.0 \%$ of the control individuals. Most of the variants resulted in loss of function of KSR2. Of the 27 variants, 23 were only found in patients with severe obesity.

KSR2 was also sequenced in 44 family members of the severely obese participants. Variants were found in 19 family members, of whom 18 were overweight or obese. However, obesity did not consistently co-segregate with variants of KSR2 within families, which suggests that other genomic variants, epigenomic events or environmental factors are also involved in the predisposition to obesity.

The team then went on to evaluate the phenotype of carriers of KSR2 mutations in 18 study participants and family members. 26 noncarriers who were matched to carriers for obesity were recruited from the local population as controls. Final height was not affected by the mutations, which suggests that KSR2 is not needed for growth. However, energy intake was increased in children, but not adults, with the mutations. Moreover, the basal metabolic rate was lower in adult carriers than in noncarriers, but was not significantly different in children. Fasting levels of insulin were much higher in carriers than in noncarriers, and mutation carriers also had impaired glucose tolerance in response to a glucose challenge. These findings, together with other clinical observations, indicate that patients without functional KSR2 have severe insulin resistance.

The researchers used $\mathrm{Ksr}^{-/-}$mice to investigate how the mutations affect energy homeostasis. The knockout mice ate more and gained more weight when fed ad libitum than did wild-type mice. Even when they were fed the same amount as wild-type mice, knockout mice gained more weight, mostly due to an increase in fat mass, which suggests that energy expenditure was reduced in the knockout mice.

An experiment that used HEK293 cells overexpressing wild-type and mutant KSR2 found that although all the mutated proteins were able to bind AMPK (the fuel sensor in the cell), some did so with reduced affinity. Further studies revealed that glucose and lipid oxidation was reduced in cells containing mutated KSR2. A separate analysis showed that the KSR2 variants disrupt signalling via the Raf-MEK-ERK pathway. "Together, these data demonstrate that, when studied in cells, KSR2 mutations are associated with disrupted fuel oxidation," write the authors. "The reduced level of fatty acid oxidation was rescued by the addition of metformin, which suggests that there might be an opportunity to develop effective pharmacotherapy to counteract the obesogenic and diabetogenic potential of KRS2 loss-of-function mutations," comments Claude Bouchard (Pennington Biomedical Research Center), who was not involved in the study.

These findings show that the development of early-onset obesity and insulin resistance is associated with rare

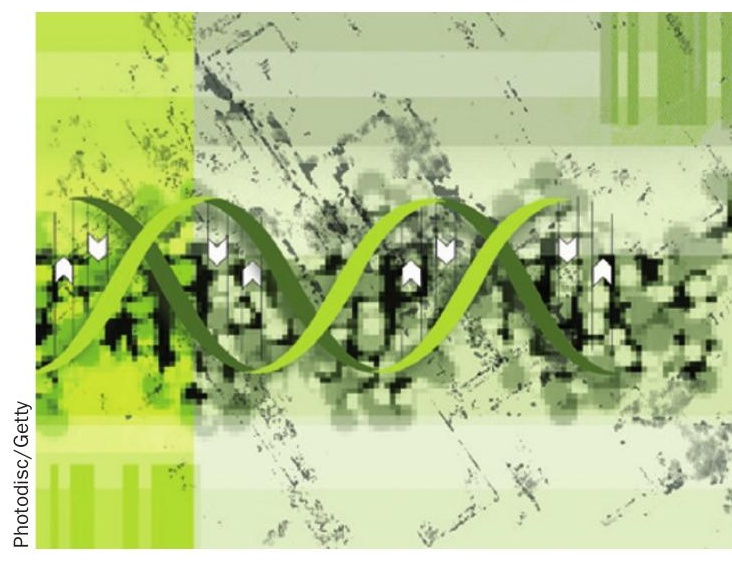

variants in KSR2 that affect its protein function. As most of the variants were found in patients with severe obesity, $\mathrm{KSR} 2$ is probably the major phenotypic determinant in these patients, in combination with other factors.

"This report, combined with the lessons from genome-wide association studies of obesity, has revealed that the biology of the predisposition to the common form of human obesity results from a relatively large number of genes, with the risk alleles having small effect sizes," says Bouchard. "The predisposition is in the end defined by the number of risk alleles carried by a given individual."

The authors of the study note that more work is required to fully understand the effects of the variants they have identified, particularly in relation to AMPK.

However, they conclude that their findings suggest that pharmacological approaches based on modulating the activity of KSR2 could be a new therapeutic strategy for treating patients with obesity and type 2 diabetes mellitus.

Claire Greenhill 